\title{
Adolescent Linkage to Care After a Large-scale Transfer From a Hospital-based HIV Clinic to the Public Sector in South Africa
}

\section{Citation}

Ramirez-Avila, Lynn, Susan Regan, Christie Cloete, Tamaryn Crankshaw, Dustin J. Rabideau, Kenneth A. Freedberg, Robert A. Parker, Rochelle P. Walensky, Elena Losina, and Ingrid V. Bassett. 2017. "Adolescent Linkage to Care After a Large-scale Transfer From a Hospital-based HIV Clinic to the Public Sector in South Africa." The Pediatric Infectious Disease Journal 36 (3): 311-313. doi:10.1097/INF.0000000000001392. http://dx.doi.org/10.1097/INF.0000000000001392.

\section{Published Version}

doi:10.1097/INF.0000000000001392

\section{Permanent link}

http://nrs.harvard.edu/urn-3:HUL.InstRepos:32072158

\section{Terms of Use}

This article was downloaded from Harvard University's DASH repository, and is made available under the terms and conditions applicable to Other Posted Material, as set forth at http:// nrs.harvard.edu/urn-3:HUL.InstRepos:dash.current.terms-of-use\#LAA

\section{Share Your Story}

The Harvard community has made this article openly available.

Please share how this access benefits you. Submit a story.

Accessibility 


\title{
Adolescent Linkage to Care After a Large-scale Transfer From a Hospital-based HIV Clinic to the Public Sector in South Africa
}

\author{
Lynn Ramirez-Avila, MD, MSc, * Susan Regan, PhD, $†$ t Christie Cloete, MBChB, $\S$ Tamaryn Crankshaw, PhD, 9

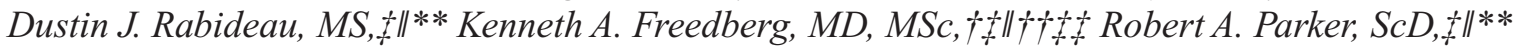

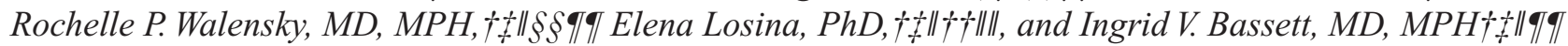

\begin{abstract}
HIV clinics formerly supported by the President's Emergency Plan for AIDS Relief are transferring patients to public-sector clinics. We evaluated adolescent linkage to care after a large-scale transfer from a President's Emergency Plan for AIDS Relief-subsidized pediatric HIV clinic in Durban, South Africa. All adolescents (11-18 years) in care at a pediatric statesubsidized, hospital-based clinic (HBC) were transferred, from May to June 2012, to government sites [primary health care (PHC) clinic; community health center (CHC); and $\mathrm{HBCs}]$ or private clinics. Caregivers were surveyed 7-8 months after transfer to assess their adolescents' linkage to care and their reports were validated by clinic record audits in a subset of randomly selected clinics. Of the $309(91 \%)$ caregivers reached, only 5 (2\%) reported that their adolescent did not link. Of the 304 adolescents who linked, 105 (35\%) were referred to a PHC, 73 (24\%) to a CHC and $106(35 \%)$ to a HBC. A total of 146 (48\%) linked adolescents attended a different clinic than that
\end{abstract}

\section{Accepted for publication June 5, 2016}

From the *Division of Pediatric Infectious Diseases and Global Health, Department of Pediatrics, University of California at San Francisco, San Francisco, California; $†$ Division of General Internal Medicine, Massachusetts General Hospital, Boston, Massachusetts; $\$$ Medical Practice Evaluation Center, Department of Medicine, Massachusetts General Hospital, Boston, Mas-

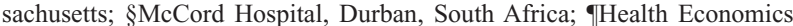
and HIV and AIDS Research Division (HEARD), University of KwaZuluNatal, South Africa; IHarvard University Center for AIDS Research (CFAR), Boston, Massachusetts; **Biostatistics Center, Massachusetts General Hospital, Boston, Massachusetts; † Departments of Biostatistics and Epidemiology, Boston University School of Public Health, Boston, Massachusetts; + Departments of Epidemiology and Health Policy and Management, Harvard School of Public Health, Boston, Massachusetts; $\S \S$ Division of Infectious Disease, Department of Medicine, Brigham and Women's Hospital, Boston, Massachusetts; ब9Division of Infectious Disease, Department of Medicine, Massachusetts General Hospital, Boston, Massachusetts; and IIIDepartment of Orthopedic Surgery, Brigham and Women's Hospital, Boston, Massachusetts.

The study protocol was approved by the McCord Hospital Research Ethics Committee (Durban, South Africa) and the Partners Human Research Committee (2012-P-001122/1, Boston, MA).

Presented in part at the 2013 ID Week, October 2-6, 2013, San Francisco CA, Abstract 4105 .

Supported in part by the Health and Human Services, Health Disparities Post Graduate Fellowship, and National Institute of Allergy and Infectious Disease: T32 AI 007433; K23 AI 068458; K24 AI062476; R01 AI058736; the Eunice Kennedy Shriver National Institute of Child Health and Human Development (NICHD) T32 HD 055148-02, the Harvard University Center for AIDS Research P30 AI060354; the National Institute of Mental Health: R01 MH090326-03S1; R01 MH073445. The content is solely the responsibility of the authors and does not necessarily represent the official views of the National Institutes of Health.

The authors have no conflicts of interest to disclose.

Address for correspondence: Lynn Ramirez-Avila, MD, MSc, Division of Pediatric Infectious Diseases and Global Health, Department of Pediatrics, Benioff Children's Hospital, University of California at San Francisco, Mission Hall, 550 16th Street, 4th Floor, Desk 4842, San Francisco, CA 94158. E-mail: lynn.ramirez@ucsf.edu.

Copyright $(2017$ The Author(s). Published by Wolters Kluwer Health, Inc. This is an open-access article distributed under the terms of the Creative Commons Attribution-Non Commercial-No Derivatives License 4.0 (CCBY-NC$\mathrm{ND}$ ), where it is permissible to download and share the work provided it is properly cited. The work cannot be changed in any way or used commercially without permission from the journal.

ISSN: 0891-3668/17/3603-0311

DOI: 10.1097/INF.0000000000001392 assigned. Thirty-five (20\%) of the 178 who linked and were assigned to a PHC or CHC ultimately attended a HBC. Based on clinic validation, the estimated transfer success was $88 \%$ (95\% confidence interval: $77 \%-97 \%)$. The large majority of adolescents successfully transferred to a new HIV clinic, although nearly half attended a clinic other than that assigned.

Key Words: PEPFAR, transfer of HIV care, South Africa, pediatric, adolescent

(Pediatr Infect Dis J 2017;36:311-313)

$\mathrm{T}_{\mathrm{p}}^{\mathrm{o}}$ o rapidly scale-up HIV services, the President's Emergency Plan for AIDS Relief (PEPFAR) initially partnered with private, hospital-based nongovernmental organizations, before a community-based, nurse-led HIV clinic model was adopted. ${ }^{1,2}$

No studies specifically assess HIV care decentralization in adolescents despite their unique care needs..$^{3-5}$ Our objective was to evaluate linkage of adolescents after a large-scale transfer from a state-subsidized hospital-based, PEPFAR-funded pediatric HIV clinic to the public sector in South Africa.

\section{MATERIALS AND METHODS}

\section{Study Site}

McCord Hospital was a state-subsidized, semiprivate hospital in Durban that became PEPFAR-funded in $2004 .{ }^{6}$ The Sinkithemba HIV Clinic at McCord provided comprehensive pediatric care $(<18$ years) by a dedicated team of nurses, physicians, counselors and social worker for a subsidized monthly fee. ${ }^{2}$ Stable adolescents were seen every 3 months. Due to loss of PEPFAR funding, the clinic closed in 2012 and all the patients were transferred to other facilities. ${ }^{6}$

\section{Sinikithemba Transfer Process}

Pediatric patients were transferred, from May to June 2012, if they presented for follow-up or a pharmacy refill. Adolescents 11-18 years who visited the clinic during this period were considered eligible.

At time of transfer, adolescents and their caregivers were counseled, provided 1 month antiretroviral therapy supply, and referred to a new clinic, based on care needs and residential address. In collaboration with Municipal and Provincial Departments of Health, patients were transferred to 3 types of government clinics: (1) primary health care (PHC) clinics that are small, nurse-run facilities; (2) larger community health centers (CHCs) that offer more medical services including occasional physician staffing and (3) hospital-based clinics (HBCs) that are physician-led and offer the most specialized HIV care, some with specific pediatric services. Few patients transferred to private clinics. Receiving clinics did not receive technical support from McCord.

\section{Data Collection}

A research assistant contacted the adolescent's caregiver via phone 7-8 months after transfer to inquire about their adolescent's 
HIV care, including which clinic they attended. Adolescents were categorized as having received care at the assigned clinic if attendance was reported at the original referral site or an alternate clinic if attendance at a different clinic was reported. After 3 failed phone attempts, a caregiver was considered unreachable. Caregivers gave verbal consent to have their adolescent's anonymized data used for research.

We selected a random sample of clinics to validate caregiver's report of the adolescent's first transfer clinic visit by examining clinic records, with the goal of validating at least $10 \%$ of visits. ${ }^{6}$ Ten clinics were selected for the validation analysis; 2 were excluded because of incomplete records. We sought all adolescents assigned to or with reported attendance at a clinic.

\section{Statistical Analysis}

Characteristics of adolescents whose caregivers were reached or not reached were compared using the Fisher's exact test or Wilcoxon rank-sum test. Linkage was defined as at least 1 HIV clinic visit since transfer. Using validation data, we estimated the overall success of transfer (Fig. 1). We used bootstrapping (10,000 iterations) to estimate the $95 \%$ confidence interval for the estimated linkage proportion. To remain conservative, we assumed that all unreached patients failed to link and drew validation probabilities from a beta distribution (1,000,000 samples) and estimated a 95\% lower bound on the linkage proportion. Statistical analyses were performed using SAS 9.4 (Cary, NC) and R version 3.1.2 (www.r-project.org).

\section{RESULTS}

\section{Transfer Cohort}

A total of 341 adolescents had at least 1 HIV clinic visit during the transfer period (Fig. 1). Median age was 13 years [interquartile range (IQR): 12-15] with 239 (70\%) adolescents $<15$ years; $173(51 \%)$ were female. The median baseline cluster of differentiation 4 (CD4) count was 219/ $\mathrm{mm}^{3}$ (IQR: 53-426) and the most recent median CD4 count before transfer was 619/ $\mathrm{mm}^{3}$ (IQR: 446-889). The majority of adolescents were on an efavirenz-based regimen $(n=253 ; 76 \%)$ and their last measured viral load was undetectable $(\mathrm{n}=259 ; 77 \%)$.

The caregivers of $309(91 \%)$ adolescents were contacted (Fig. 1); 42\% ( $\mathrm{n}=129)$ were the biological mother. Adolescents whose caregiver was reached had a significantly higher median most recent CD4 (637 vs. $480 / \mathrm{mm}^{3}, P=0.03$ ), and were more likely to have an undetectable viral load when last measured (79\% vs. $60 \%, P=0.04)$ and to be on an efavirenz-based regimen $(77 \%$ vs. $60 \%, P=0.04)$.

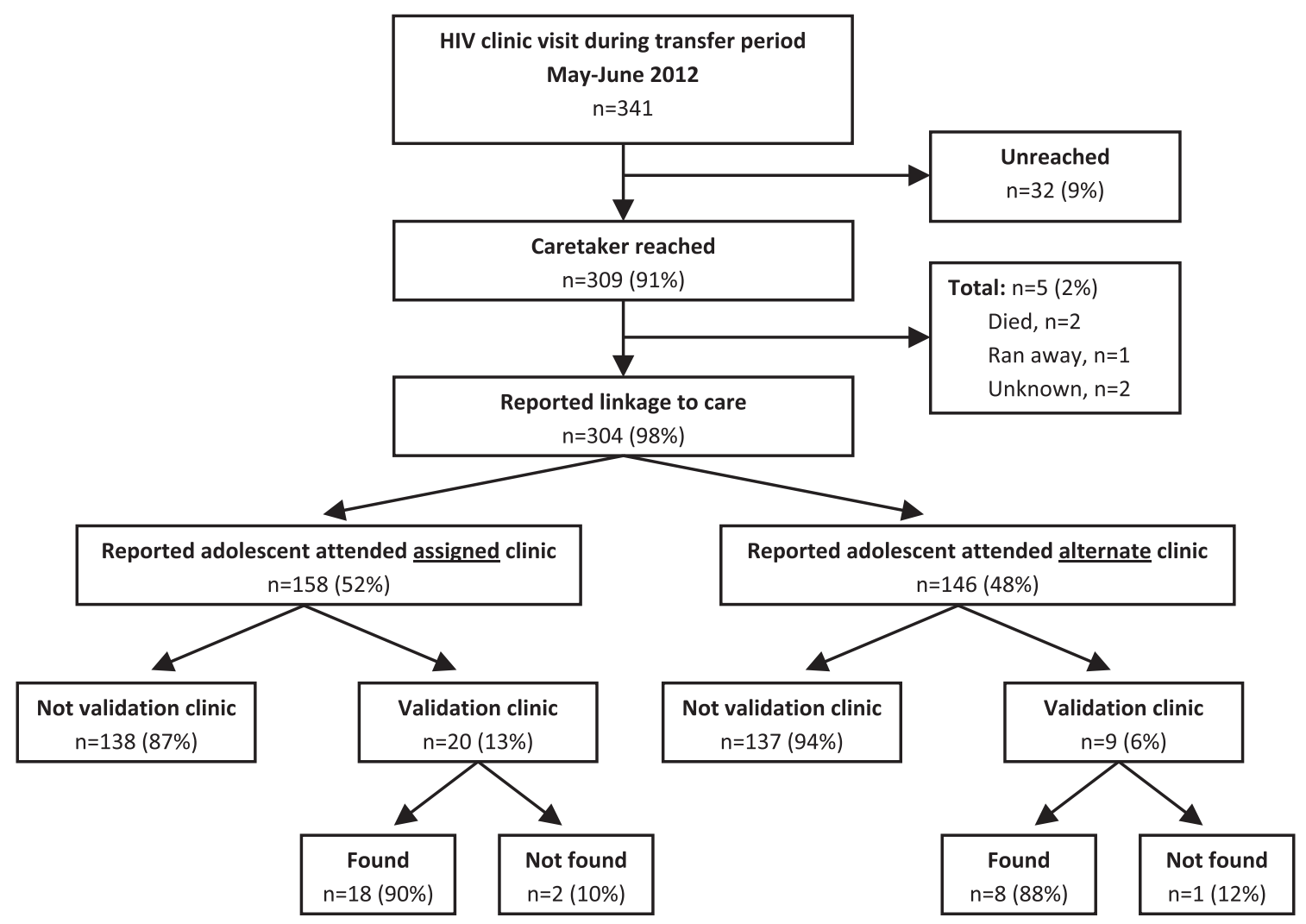

FIGURE 1. Adolescent HIV-infected transfer cohort, caregiver reported linkage to care and validation. This flow diagram shows the adolescent HIV-infected cohort that was transferred from a semiprivate, PEPFAR-subsidized clinic to the public sector. We display the proportion reached for telephone survey, the proportion that reported at least 1 clinic visit since transfer, and the proportions found at validation clinics. Estimated success of transfer: $\left[F_{\mathrm{A}}\left(R_{\mathrm{A}}\right)+F_{\mathrm{SR}}\left(1-R_{\mathrm{A}}\right)\right] \times L=\left[\left(\frac{18}{20}\right)\left(\frac{158}{304}\right)+\left(\frac{8}{9}\right)\left(\frac{146}{304}\right)\right] \times\left(\frac{304}{309}\right)=0.8802=88 \%$, where $F_{\mathrm{A}}$ is the proportion of patients found at the assigned clinic during validation; $F_{S R^{\prime}}$ the proportion found at the alternate clinic during validation; $R_{A^{\prime}}$ the overall proportion of patients who reported attending the assigned clinic and $L$, the proportion of patients reporting linkage to care among those contacted. 


\section{Caregiver Reported Linkage to Care}

The majority of contacted caregivers reported their adolescent had at least 1 clinical visit since transfer $(n=304 ; 98 \%$; Fig. 1). The median number of reported visits since transfer was 5 (IQR: 5-6). Of the 5 adolescents who did not link, 2 reportedly died, while another ran away.

Adolescents whose caregiver reported linkage were assigned to PHCs $(n=105 ; 35 \%)$, CHCs $(n=73 ; 24 \%)$ or HBCs $(n=106$; $35 \%)$. Nineteen $(6 \%)$ were assigned to private clinics; clinic type could not be determined for 1 adolescent. Among the 304 who linked, $158(52 \%)$ transferred to the assigned clinic and $146(48 \%)$ to an alternate clinic. Of the 178 adolescents who linked and were assigned to a PHC or CHC, 35 (20\%) attended a HBC instead. Including those who presented to their assigned and to an alternate clinic, a total of $141(46 \%)$ of the linked adolescents went to a HBC.

\section{Validation of Caregiver Reported Clinic Visits}

Of 8 validation clinics, 1 was a $\mathrm{CHC}$ and 7 were PHCs. There were 20 adolescents assigned to and whose caregiver reported attendance at 1 of the validation clinics; an additional 9 adolescents were assigned to nonvalidation clinics, but their caregiver reported attendance at a validation clinic. The $29(9 \%)$ adolescents in the validation cohort did not differ from the rest of the cohort by age, sex, most recent CD4 and proportion with undetectable viral load $(P>0.05)$. The validation cohort had lower median baseline CD4 compared with the rest of the transfer cohort (124 vs. $233 / \mathrm{mm}^{3}, P=0.03$ ).

Of 20 adolescents with reported assigned clinic attendance, record was found for $18(90 \%)$. Of 9 adolescents with reported alternate clinic attendance, record was found for 8 adolescents $(89 \%)$. Based on this validation and the overall proportion of adolescents who reported attendance to the assigned clinic (52\%), the estimated success of transfer was $88 \%$ (95\% confidence interval: 77\%-97\%; Fig. 1). Under the conservative assumption that all unreached patients failed to link, we are $95 \%$ confident that the true linkage is at least $69 \%$.

\section{DISCUSSION}

We report the mandatory transfer of an HIV-infected adolescent cohort from a PEPFAR-funded, HBC to the public sector. Of $91 \%$ of caregivers reached after transfer, 98\% reported that their adolescent attended an HIV clinic. For adolescents whose caregivers were reached, the estimated overall success of transfer was $88 \%$ based on clinic validation. Many adolescents (48\%) attended an alternate clinic.

Linkage to an HIV clinic for this transferred adolescent cohort was high, likely due coordinated efforts involving clinic leadership and the pediatric team at Sinikithemba. The estimated linkage of adults transferred from the same clinic was also high at $82 \% .{ }^{6}$ South Africa has proposed multiple strategies to scale up its HIV program, including supporting HIV care at PHC, nurse-run clinics. ${ }^{2}$ Several adult and pediatric studies have reported favorable outcomes, including loss to follow-up and virologic suppression, in nurse-run PHC programs compared with $\mathrm{HBCs}^{7-9}$ although some have reported higher mortality at local clinics. ${ }^{10}$ In the current study, a large number of adolescents were referred to and attended a HBC although they were on first-line therapy with high CD4 counts before transfer.

Caregivers surveyed at least 7 months after transfer reported their adolescent had a median of 5 clinic visits. However, to have a complete understanding of linkage to care after a mandatory transfer, longer follow-up time and clinic validation is needed to assess retention in care, adherence and treatment interruption.

We do not know why some adolescents referred to PHCs and CHCs ultimately attended HBCs; possibilities include geographic convenience, familiarity with $\mathrm{HBC}$ structure, availability of pediatric drug formulations, being turned away by the $\mathrm{PHC}$ or $\mathrm{CHC}$ and caregiver perception of better quality care. This may also reflect that certain
HBCs are aware that HIV-infected adolescents have specific care needs given lower adherence and virologic suppression, higher rates of virologic rebound and specific psychosocial and developmental needs. ${ }^{4,5}$

Our study has a number of limitations. Adolescents who did not present for care during the transfer period were not included in the study cohort and may have been referred after the clinic closed. Approximately $9 \%$ of the caregivers were not reachable via phone, and we relied on caregiver report for linkage to care. We did not elicit the transfer and new clinic experiences of the caretakers and adolescents. The clinic validation process varied at the target sites because of different medical record systems and methods of patient identification. This cohort may not be representative of patients seeking care in government clinics because Sinkithemba patients paid a subsidized fee and were maintained in pediatric care until 18 years, beyond the usual age of transition to adult services in some clinics.

We found that most adolescents had a first HIV clinic visit after the rapid closure of a PEPFAR-subsidized HIV clinic. The largest number of adolescents with at least 1 reported clinic visit attended a HBC. Ongoing efforts to successfully decentralize the care of adolescents are needed as PEPFAR support of HIV programs transitions to the South African government.

\section{ACKNOWLEDGMENTS}

We gratefully acknowledge the hard work of Siphesihle Sithole in contacting the patients transferred from Sinikithemba. We thank Drs. Helga Holst, Peninah Thumbi and Kristy Nixon at McCord for providing strong leadership during a time of transition. We also thank Tessa Govender and Janet Giddy. Finally, we acknowledge Penny Dladla, eThekwini District Health Services Manager, and Elizabeth Lutge, Chairperson of the Health Research Committee of the KwaZulu-Natal Provincial Department of Health, for facilitating access to community-based clinics for visit validation.

\section{REFERENCES}

1. Larson E, O'Bra H, Brown JW, et al. Supporting the massive scale-up of antiretroviral therapy: the evolution of PEPFAR-supported treatment facilities in South Africa, 2005-2009. BMC Public Health. 2012;12:173.

2. Department of Health, Republic of South Africa, Guidelines for the Management of HIV in Children. 2nd ed. 2010. Available at: http://www. sahivsoc.org/upload/documents/Guidelines_for_Management_of_HIV_in_ Children_2010.pdf. Accessed June 20, $201 \overline{5}$.

3. Pettifor AE, Rees HV, Steffenson A, et al. HIV and Sexual Behavior Among Young South Africans: A National Survey of 15-24 Years Olds. Johannesburg: Reproductive Health Research Unit, University of Witwatersrand; 2004. Available at: https://kaiserfamilyfoundation.files.wordpress.com/2013/01/ hiv-and-sexual-behaviour-among-young-south-africans-a-national-surveyof-15-24-year-olds.pdf. Accessed June 20, 2015.

4. Agwu AL, Fairlie L. Antiretroviral treatment, management challenges and outcomes in perinatally HIV-infected adolescents. J Int AIDS Soc. 2013;16:18579.

5. Cowan F, Pettifor A. HIV in adolescents in sub-Saharan Africa. Curr Opin HIV AIDS. 2009;4:288-293.

6. Cloete C, Regan, S, GIddy, J, et al. The linkage outcomes of large-scale, rapid transfer of HIV-infected patients from hospital-based to communitybased clinics in South Africa. Open Forum Infectious Diseases. 2014;1:1-9.

7. Brennan AT, Long L, Maskew M, et al. Outcomes of stable HIV-positive patients down-referred from a doctor-managed antiretroviral therapy clinic to a nurse-managed primary health clinic for monitoring and treatment. AIDS. 2011;25:2027-2036.

8. Long L, Brennan A, Fox MP, et al. Treatment outcomes and cost-effectiveness of shifting management of stable ART patients to nurses in South Africa: an observational cohort. PLoS Med. 2011;8:e1001055.

9. Bock P, Boulle A, White C, et al. Provision of antiretroviral therapy to children within the public sector of South Africa. Trans R Soc Trop Med Hyg. 2008;102:905-911.

10. Massaquoi M, Zachariah R, Manzi M, et al. Patient retention and attrition on antiretroviral treatment at district level in rural Malawi. Trans $R$ Soc Trop Med Hyg. 2009; 103:594-600. 